Reinfelder, Fritz, Ein Fall von beiderseitiger Verdoppelung der Nieren und Ureteren, zugleich eine Zusammenstellung einiger Anomalien der Niere, des Nierenbeckens und der Ureteren überhanpt. Inaug.-Diss., München 1905.

Junge. Rudolf, Ein seltener Fall von Uretercyste. Inaug.-Diss., Berlin 1904 .

\title{
XVIII.
}

\section{Zur Kenntnis der Rhabdomyome des weiblichen Geschlechtsorgans.}

\author{
Von \\ Dr. F. Berka, Prosektor in Olmütz. \\ (Hierzu Taf. XI.)
}

A. G., 18 Jahre alt, Tochter eines Knopfmachers; war stets gesund und bot für das Auftreten von Geschwälsten keine Anhaltspunkte. Seit dem 16. Jahre Eintritt der Menstruation: Menses 3- bis 4 tägig, in fünfwöchentlichen $\mathrm{Zwischenräumen.} \mathrm{Niemals} \mathrm{zeigten} \mathrm{sich} \mathrm{unregelmäßige} \mathrm{oder}$ abnolme Blutungen. Im Juni 1905 bemerkte das Mädchen ejnen braunen, wässerigen Ausfuß ans dem Genitale, der ursprünglich minimal und nur durch das Auftreten von Flecken in der Wäsche kenntlich war, sich jedoch später vermehrte. Nach etwa einem Monat verspürte sie das Gefühl eines Fremdkörpers in der Schamspalte und konnte mit eigenen Fingern einen kleinhaselnuBgroBen Tumor in der Vulva tasten. In der nachfolgenden Zeit vermehrte sich der Ausfuß, wurde profus und übelriechend; auch der Tumor vergrößerte sich und senkte sich tiefer vor die Schamspalte, was die Patientin nötigte, anfangs September 1905 Spitalshilfe aufznsuchen. Blutungen oder Schmerzen hatten sich nie eingestellt.

Bei der Aufnahme war der auffälligste Befund das Vorhandensein eines stark belegten, derben Tumors, welcher dem Genitale vorgelagert war. Seine längliche Form, Größe sowie die Vorlagerung sollen an das Prolabieren eines kindlichen Fußes bei Steißlage erinnert haben; mit dem inneren Genitale hing die Geschwulst mittels eines kindesschenkeldicken Stieles zusammen. Es zeigte sich, daß dieser der stark gedehnten und in die Länge gezogenen Cervix uteri entsprach, an deren unterstem Pole sich die Geschwulst inserierte. Durch letztere hindurch, an der Unterfläche seiner vorderen Hälfte ausmïndend, verlief der Cervikalkanal. Der Uterus klein, sowie die Adnexa ohne Besonderheiten. Die Scheidengewölbe in gewöhnlicher Höhe, so daß der Situs der Beckeneingeweide ungefähr Schema Fig. 1, Taf. XI entsprach. 
In der gynäkologischen Abteilung der Gebäranstalt in Brünn (Direktor Prof. Dr. H. Riedinger, dessen Liebenswürdigkeit ich das anatomische Präparat verdanke) wurde der Stiel der Geschwulst mittels Paquelin durchgetrennt und der Tumor mir behufs diagnostischer Untersuchung übermittelt.

Nach Durchsicht der mittels Gefriermikrotom hergestellten und mit Hämatoxylin gefärbten Präparate wurde vorläufig die Geschwulst, deren alveolärer Bau einem Karzinom nicht unähnlich sah, als Sarkom von alveolärem Bau agnosciert. Mit Rüeksicht anf diesen Befund wurde die vaginale Totalexstirpation des Uterus und Adnexe an der A. G. vorgenommen, deren Untersuchung nichts Krankhaftes ergab. Die Kranke wurde nach glatter Wundheilung entlassen.

Über den weiteren Decursus morbi konnte ich leider keine detaillierten Angaben erhalten; man teilte mir mit, daß sie binnen eines Vierteljahres nach der Entlassung ans dem Krankenhause im Elternhause gestorben sei. Es ist zweifellos, daB sie ihrem im Krankenhause beobachteten Leiden erlegen ist.

Die bei der ersten Operation gewonnene Geschwulst (Fig. 2, Taf. XI) bot im gehärteten Zustande (Formalin) nachstehende Verhältnisse:

Die Form derselben kann als länglich walzenförmig mit mehreren rundlichen, knolligen Vorragungen bezeichnet werden; sie ist $8 \mathrm{~cm}$ lang, von $4 \frac{1}{2} \mathrm{~cm}$ Breiten- und $4 \frac{1}{2} \mathrm{~cm}$ Höhendurchmesser. An ihrer oberen Fläche nach vorn von der Mitte befindet sich die schwarzbraun verschorfte, $2 \frac{1}{2} \mathrm{~cm}$ im Durchmesser betragende Abtrennungsstelle vom Uterus, die im gehärteten Präparat infolge rascher Retraktion der ursprünglich oedematösen Stielinsertion trichterförmig eingezogen ist. Nach hinten angrenzend findet sich eine länglichrundliche, quergestellte Vorwölbung, welche mit Rïcksicht auf ihre Form und ihre makroskopisch bemerkbare Schleimhautbedeckung (sie ist mit weiß sich hervorhebendem und leicht prominentem Epithelbelag gegenüber den uibrigen, keine Schleimhaut aufweisenden Geschwulstanteilen scharf gekennzeichnet) an die Form einer Muttermundslippe erinnert. Von dieser Vorwölbung läßt sich die Epithelbedeckung teils als Umgrenzung des Abtrennungsschorfes, teils in Form vereinzelter, wie versprengter kleiner Inseln verfolgen, welche einer halbkugeligen Prominenz am hinteren oberen Pole der Geschwulst stellenweise aufsitzen. Die übrige Oberflächenkonfiguration ist die mehrerer, bis haselnußgroher, halbkugeliger, graurötlicher Knollen (etwa 8), welche oberflächlich ziemlich glatt sind und nirgends Epithelbelag aufweisen.

Vom Grunde des Verschorfungstrichters läßt sich nach der Unterfläche des Tumors der überall wegsame und zahlreiche Längsfalten bergende Cervikalkanal verfolgen, welcher zwischen den halbkugeligen Erhabenheiten ausmündet. Seine Wandung ist glatt, leicht buchtig.

Am Durchschnitte ist die Geschwulstmasse grauweiß, von zahlreichen feinen, aus dem Innern gegen die Oberfläche ausstrahlenden weißlichen 
Zügen durchsetzt. Etwa $1 \mathrm{~cm}$ nach hinten vom Cervikalkanal findet sich im Tumorgewebe ein glattwandiger, mit klarer Flüssigkeit gefüllter, kugeliger Hohlraum, $\frac{3}{4} \mathrm{~cm}$ im Durchmesser.

Die Konsistenz des frischen Tumors war eine derbe.

Der: nachträglich exstirpierte Uterus, welcher sich nach der Geschwulstentfenung bereits vollends verkürzt hatte, war $7 \mathrm{~cm}$ lang, im Fundusteile $4 \frac{1}{2} \mathrm{~cm}$ breit, Wandung bis $1 \frac{1}{2} \mathrm{~cm}$ dick. Die Innenfläche blab, glatt, ohne Spuren von Geschwulstwucherung. Cavum uteri etwa $3 \mathrm{~cm}$ lang, durch den deutlich abgesetzten inneren Muttermund von dem etwa $2 \mathrm{~cm}$ langen Cervixteile getrennt. Die Schleimhant desselben mit ausgebildeten Plicae palmatae, blaß, die Wandung unverändert. Auf dem unteren Pole der Cervix die granulierende Abtrennungsfläche vom Tumor, welche ringförmig ist, von $3 \frac{1}{2} \mathrm{~cm}$ im Durchmesser, wovon ungefähr ein Drittel dem Uterus, zwei Drittel der Scheide angehören. Der Scheidenanteil ohne Veränderungen. Ovarien mit wenigen Kerben, Tuben und ïbrige Adnexa normal.

Nachdem es nach der Lokalisation und der vorläufigen diagnostischen Durchsicht wahrscheinlich wurde, daß es sich im vorliegenden Falle nicht um ein gewöhnliches Sarkom, sondern vielleicht urm einen der seltenen Vaginal- bzw. Portiotumoren handle, welche durch ihr Einreihen in das System der Mischgeschwülste, ihren Gehalt an fremden Gewebsbestandteilen besonderes Interesse erheischen, wurde der bereits seit längerer Zeit fixierte Tumor einer genauen mikroskopischen Untersuchung unterzogen.

Bekanntlich waren die Gesehwälste des weiblichen Genitales nach den Nierengeschwälsten die hänfigste Fundstätfe von heterologen Geweben, unter welchen quergestreifte Muskulatur und Knorpel besonders zu nennen sind. Die von Spiegelberg. beschriebenen, von Weigert untersuchten traubenmolenartigen Sarkome der Cervix uteri, welche eine relativ gut abgegrenzte Geschwulstgruppe zu bilden scheinen, pflegen nicht selten beides zu enthalten; bei den Sarkomen der Scheide haben Hauser ${ }^{1}$ ), Kolisko $0^{2}$ ) und Kaschewarowa-Rudnewa ${ }^{3}$ ) quergestreifte Muskelfasern nachgewiesen. Ohne auf die Literatur dieses

1) Hauser, Beiträge zur Genese des primären Scheidensarkoms. Dieses Archiv, Bd. 88, 1882.

2) Kolisko, Das polypöse Sarkom der Vagina im Kindesalter. Wiener klin. Wochenschr. 1889, Nr. 6.

3) Kaschewarowa-Rudnewa, Primäres Scheidensarkom. Dieses Archiv, Bd. 54, 1872. 
Kapitels näher einzugehen, will ich nur orientierend bemerken, daß von den zwei geschiedenen Gruppen dieser Geschwülste der einen, i. e. den Sarkomen der Cervix uteri, häufig Bildung blasiger, beeren- oder traubenmolenartiger Massen (Sarcoma hydropicum papillare - Spiegelberg) eigen ist; solche Gebilde bestehen aus einem schleimgewebeartigen Gewebe, in welchem Rundzellenhaufen sowie Spindelzellenstränge vorkommen, und enthalten nicht selten quergestreifte Muskulatur sowie Knorpelherde. Die zweite Gruppe sind die sarkornatösen Tumoren der Scheide (namentlich bei Kindern). Dieselben sind von schleimpolypenartigem Aussehen (polypöses Sarkom - $\mathrm{K}_{0}$ lisko), und ähnelt ihr feinerer Bau im großen dem der Cervixtumoren, wobei allerdings die quergestreiften Muskelfasern relativ seltener, Knorpelzellen nie gefunden wurden. Von dieser zweiten Gruppe will man noch die Sarkome der Scheide der Erwachsenen, zu welchen auch die der Portio zu zählen wären, trennen (Rosthorn). Wilms ${ }^{1}$ ) hat die nahe Verwandtschaft dieser zusammengesetzten Tumoren (,Mischgeschwülste“) kritisch beleuchtet und eine einheitliche Entstehungshypothese für dieselben gegeben. - Dies waren die Gesichtspunkte, die uns zu einer genauen mikroskopischen Untersuchung unseres Präparates bewogen und eine komplizierte Zusammensetzung desselben vermuten ließen, trotzdern die vorläufige Untersuchung (Hämatoxylin-, Hämatoxylin-Eosinfärbung) einfaches Alveolärsarkom und zunächst keine fremdartigen Elemente entdeckte, und die makroskopische (derbe) Beschaffenheit schwerlich in den Rahmen der traubenartigen Geschwülste eingezwängt werden konnte.

Es wurden aus mehreren Stellen der Geschwulst Stückchen excidiert, die im mikroskopischen Präparate nachstehende Bilder gaben:

1. Die mit Epithel bedeckte Partie nach hinten vom Abtrennungsschorfe: das Oberflächen-Plattenepithel wohl ausgebildet, die Zellen der obersten Lagen in Verhornung; die tiefsten Epithelschichten zeigen hie und da vereinzelte Leukocyten. Unter dem Epithel ein faseriges, relativ zellarmes Stroma, welches in den zwischen das Epithel sich einschiebenden

1) Wilms, Die Mischgeschwülste. Berlin und Leipzig, 1902. 
Papillen Gefäßschlingen, im übrigen Gefäße meist kleineren Kalibers enthält. In diesem Stroma befinden sich deutlich abgegrenzte Nester oder Säulen von großen, rundzellenähnlichen Zellen, deren lebhaft tingierte, rundliche oder unregelmäßig geformte Kerne im Durchschnitt 8 bis $10 \mu$ betragen (Fig. 3, Taf. XI). Nach dém Innem zu werden die Zelinester größer, liegen dichter; schließlich konfluieren sie, lassen die alveoläre Anordnung kaum erkennen und stellen ein zellreiches, einem Rundzellensarkom ähnliches Gewebe dar, in welchem ein faseriges Stroma nicht mehr bemerkbar ist. An van Gieson-Präparaten sieht man in den Zellnestern sehr spärlich größere, bis $30 \mu$ im Durchmesser betragende, durch Pikrinsäure hellgelb gefärbte rundliche oder polygonale Zellleiber mit granuliertem Protoplasma; welche entweder mehrere Kerne oder einen großen Kern mit großen Kernkörperchen enthalten. Oedematöses Gewebe bzw. mit Endothel ausgekleidete Hohlräume, wie in den Cervizsarkomen häufig beschrieben, finden sich nicht vor.

2. Geschwulstgewebe entsprechend dem vorderen Pole der Geschwalst: die glatte Oberfläche' trägt kein Epithel; ihre oberflächlichen Schichten bestehen aus Bindegewebsstroma, welches kleinzellig infiltriert ist and weiter nach dem Inneren zellreiches, an Granulationsgewebe erinnerndes Gewebe mit reichlichen Gefäßen zeigt. In diesem liegen einige Quexschnitte von wohlerhaltenen Schleimdrüsen vom Typus derer des Cervix mit einreihigem, hohem Cylinderepithel. Außerdem finden sich hier noch rundliche Gebilde von ungefähr demselben Durchmesser wie die Drüsen; dieselben sind bindegewebig mehr oder weniger deutlich abgekapselt und bestehen aus großen, epithelartigen Zellen, welche konzentrisch geschichtet diese runden Gebilde ausfüllen and bläschenförmige, längliche Kerne mit deutlichen Kernkörperchen besitzen. In ihrer Peripherie finden sich spärlich Leukocyten, im Zentrum nicht selten ein mit roten Blutzellen gefüllter Gefäßquerschnitt. Nach innen von diesen Schichten, welche dem Schleimhautrest des Uterus entsprechen, liegt das Tumorgewebe. Dasselbe besteht hier nicht wie bei 1 aus gesonderten Zellnestern und Stroma, sondern aus sich verschiedenartig verwebenden Lüngs-, Quer- und Schieffaserzïgen mit zahlreichen größeren Gefäßen. Bei nach van Gieson gefärbten Präparaten zeigt sich durch lebhafte rote (Säurefuchsin) Tinktion, daß ein Teil dieser Fasern fibrillärem Bindegewebe angehört, ein anderer mit ihm sich verwebender, mehr zellreicher Anteil gelblichbraun gefärbt erscheint. Bei stärkerer Vergrößerung zeigt sich hier ein buntes Durcheinander von roten Bindegewebsfibrillen, schwarz tingierten Zellkernen, die wie sub 1 anssehen, oder aber kleiner und Jänglicher, öfters auch elliptische Form mit deutlichen Kernkörperchen zeigen, ferner von gelben Fasern, welche zum größten Teile längsfibrilliert erscheinen und an einzelnen Stellen auch Querstreifung deutlich erkennen lassen. Mit Rücksicht darauf dokumentiert sich unsere Geschwulst als quergestreifte Muskelfasern enthaltend denn dahin gehörig mußte man diese Fasern mit Rücksicht auf ihr morphologisches Verhalten ansehen. 
Weiter nach der Tiefe zu sind die einzelnen Gewebselemente noch deutlicher zu trennen, indem die Bänder der quergestreiften Muskeln breiter, isolierter verlaufen und sich vom Bindegewebe und Tumorzellen schärfer abheben (Fig. 5, Taf. XI). Zuinnerst liegen schließlich mehrere Lagen von parallel verlaufenden Zügen glatter Muskelfasern, mit Bindegewebszügen abwechselnd, die sich von den quergestreiften leicht durch das Fehlen der Fibrillierung, ferner durch mehr homogen braunes, wachsartiges Aussehen und Anwesenheit dünner, längerer, stäbchenförmiger Kerne unterscheiden.

3. Partie aus dem hinteren Geschwulstpole weist in den oberfächlichen Schichten ähnliche Verhältnisse auf wie sub 2, nur finden sich keine Drüsen, und anch die dort erwähnten epithelartigen großen Zellen kommen nur vereinzelt vor. Nach innen $z u$ ist das Bild von dem zweiten insofern verschieden, als hier auch große Gruppen von Rundzellen vorkommen, welche hie und da lichte Stellen mit auseinandergewichenen Zellen aufweisen. Bei näherem Znsehen und starker Vergrößerung bemerkt man, daß auch hier zwischen diesen Zellen spärlichere Züge aus gelbgrünlicher, spinnwebartiger Masse verlaufen, in welchen man nach längerem Suchen Querstreifung finden kann. Auch sieht man rundliche Querschnitte von solchen Gebilden, die granuliert sind.

4. Das Wandgewebe des im Innern der Geschwulst befindlichen kugeligen Cystenraumes bestand zunächst aus einer einfachen Lage von kubischen Zellen mit rundlichen, bläschenförmigen Kernen. Das darunterliegende Bindegewebe grenzte sich gegen dieses Epithel mit einer Lage vollkommen abgeplatteter Bindegewebszellen ab. Dieses Verhalten, welches schön bei Cervixdrüsen zu sehen ist, sowie die Epithelform der Zellen ließen die Annahme naheliegend erscheinen, daß die Cyste einer Drüsenerweiterung ihr Entstehen verdankt. Unter der Oberfläche lagen Züge von glatter Muskulatur und lockerem Bindegewebe, zunächst konzentrisch alternierend, später unregelmäßiger und mit größeren Gefäßen untermischt. Quergestreifte Muskelfasern konnten hier nicht nachgewiesen werden.

5. Der von der Geschwulst eingeschlossene Cervixanteil: An der buchtigen Cervixinnenfläche liegt eine einfache Lage von Cylinderzellen. An diese unmittelbar angrenzend befinden sich Trmorzellen, in lockere Nester gewebt, so daß das Gefüge hier einem Zwischenstadium zwischen 1. und 4. entspricht. Von Tumornestern eingeschlossen sind auch Querschnitte von Cervixdrüsen. Elemente von quergestreifter Muskulatur sind sehr selten, und zwar als rundliche Querschnitte, vertreten. Dagegen finden sich reichlich gröbere Bündel von glatten Muskelfasern nach innen vom Tumorgewebe.

Nach den beschriebenen Typen gebaut waren auch die sonstigen anderen Stellen im Tumor entnommenen Stï̈ckchen. Die verschorfte Partie zeigte ein dem Myometrium entsprechendes Bild (glatte Muskelfasern und Bindegewebe ohne Elemente, welche dem Tumor anzurechnen wären ein Beweis, daB im Gesunden amputiert wurde). 
Von dem nachträglich exstirpierten Uterus wurde der Cervikalanteil untersucht. Derselbe zeigte gewöhnliche Verhältnisse der Schleimhant, sowie der übrigen Wand, die Drüsen waren reichlich, die oberflächlichen Schleimhautlagen etwas lockerer gewebt, die Gefäße erweitert, was vielleicht mit der vorher bestandenen Stauung im Stiele der Geschwulst in Verbindung zu bringen wäre.

Wenn wir auf Grund dieser Befunde den Bau der Geschwulst näherer Betrachtung unterziehen, konstatieren wir das Vorkommen mehrerer Gewebsarten. Von verschiedenen Autoren wurden bisher als die, solche Tumoren des weiblichen Geschlechtsschlauches (Uterus und Vagina) zusammensetzenden Gewebe beschrieben: Sarkomzellen vom Charakter der Rundund Spindelzellen mit lockerem Stroma, Myxomgewebe, glatte Muskulatur, seltener quergestreifte Muskelfasern, Fettgewebe, Epithelzellenhaufen. Bei den Cervixtumoren kommt noch Knorpel dazu; häufig beobachtet man Drüsen, die nur ganz vereinzelt als dem Tumor angehörig, im allgemeinen jedoch als Reste des ursprünglichen, schon vor der Geschwulstwucherung bestehenden Drüsenkörpers der Cervix aufgefaßt werden. Zur Erklärung der Genese der zusammengesetzten Tumoren wurde teils die Cohnheimsche Keimversprengung, teils Gewebsmetaplasie herangezogen, während Wilms und nach ihm andere bei den Mischgeschwülsten eine kongenitale Ursache annehmen, einen mesodermalen Keim, der auf einer sehr frühen embryonalen Entwicklungsstufe zurückgeblieben ist, und der erst postembryonal durch sein Wuchern und Differenzieren die Geschwulstbildung verursacht.

Quergestreifte Muskulatur wurde kaum in der Hälfte der Fälle konstatiert, und fanden sich auch die Muskelfasern meist relativ spärlich im Geschwulstgewebe (so z. B. bei Hauser nur an zwei Stellen im Geschwulstinnern). Ihr Aussehen wird verschieden geschildert. Kolisko erwähnt drei Formen: teils glatten Muskelfasern ähnelnde, jedoch mit breiteren Kernen ausgestattete Zellen mit deutlicher Querstreifung; dickere, bandförmige, schön fibrillierte, wenig quergestreifte Gebilde, und schließlich spindelige Riesenzellen mit quergestreiftem Plasma. Nach diesen Typen Iassen sich die Muskelfasern der sonstigen Fälle sowie anch des unseren einteilen, wobei nicht alle Arten 
gleichmäßig verteilt, sondern einige mehr vorherrschen, andere fehlen können. Unsere Nuskelzellen entsprechen fast ausschließlich der zweiten Art Koliskos-Bänder von Fibrillen. Die Länge derselben ließ sich stellenweise bis zu $500 \mu$ verfolgen, ihre Breite kann im Durchschnitte mit $20 \mu$ angenommen werden. Dieselben verlaufen selten ganz gerade, sondern sind meist wellig geschlängelt. Auch ihre Kontur ist nicht ganz regelmäBig, sondern besitzt hie und da einzelne buckelartige Vorsprünge. Diese Bänder zeigen im Schnitte niemals die dichte, kompakte Beschaffenheit wie die normal entwickelte Muskulatur; vielfach erscheinen sie nur als Bündel von Längsfibrillen, welche enger oder weiter voneinander lagern, event. auseinander weichen, und sich schließlich ganz voneinander entfernen, so daß sie einzeln verlaufen können. Es scheint, daß das zwischen den Sarkomzellen in der Umgebung der Muskelbänder sichtbare feine Fasernetz zum großen Teile solchen Fibrillen angehört, trotzdem eine Querstreifung an ihm nur selten nachzuweisen ist.

Uberhaupt ist die Querstreifung der Muskelzellen in solchen Geschwülsten verschieden ausgeprägt und steht weit hinter der entwickelten Muskulatur zurück; dagegen ist die Längsfibrillierung bei unserer und den meisten anderen auffallend dentlich. Die Querstreifung stellt sich dar als quere Striche, die den Fibrillen aufliegen und den Bändern ihr charakteristisches Aussehen geben; dieser Grad ist jedoch selten, häufiger sieht man eine gleichmäBige quere Körnelung der Fibrillen, entweder die ganze Dicke des Bandes oder nur seine Randzone betreffend, die undeutlich bis unsichtbar werden kann. Dies erfolgt streckenweise, indem quergestreifte Fibrillenpartien mit nicht quergestreiften abwechseln. Bei letzteren schützt außer ihrer Verbindung vorzugsweise die fibrilläre Beschaffenheit, ihre gelbgrünliche Tinktion mit Pikrinsäure vor Irrtümern.

Die Frage des spezifischen Nachweises von quergestreiften Muskelfasern in solehen Tumoren wurde vielfach diskutiert. Kolisko nimmt an, daß, nachdem er in jedem der von ihm untersuchten drei Fälle von Vaginalsarkomen der Kinder solche fand, während von den übrigen Beobachtern ähnliche Angaben nur Hauser notiert, daß man vielleicht öfters in diesen 
Tumoren quergestreifte Muskelfasern übersehen hat, welche man wahrscheinlich bei größerer Sorgfalt auffinden würde. Als Momente, welche das Auffinden begünstigen, seien genannt: möglichst frische Untersuchung der Objekte, da durch längeres Aufbewahren in den Konservierungs- oder Fixationsflüssigkeiten die Querstreifung leide, Untersuchung bei starker Vergrößerung bezw. Immersion. Als beste Färbung hat Kolisko Pikrokarmin empfohlen, wobei an den gelb gefärbten Fasern die Querstreifung am deutlichsten vortreten soll. Wenn auch die Annahme Koliskos betreffs Übersehens von Querstreifung zurückgewiesen wurde, und man auch für alle Tumoren dieser Art, wie Wilms zeigte, dieselbe nicht für unbedingtes Erfordernis ansieht, da bei der Differenzierung der mesodermalen Zelle im Tumor nicht quergestreifte Muskelfaser jedesmal zur Entwicklung zu gelangen braucht, so möchte ich nach Erfahrungen mit meinem Falle Kolisko nicht ganz unrecht geben. Ich hatte bei der ersten Untersuchung an formolfixierten, mit Hämatoxylin, Hämatoxylin-Eosin-Präparaten umsonst längere Zeit nach quergestreiften Elementen gesucht, und auch Färbung mit Pikrokarmin führte mich nicht zum Ziele (wohl wahrscheinlich wegen wechselnder Qualität der Pikrokarminlösungen), so daß ich bereits angenommen hatte, daß auch in meinen Präparaten quergestreifte Elemente nicht vorhanden seien. Als jedoch nach Monaten zufällig die Weig ertsche Modifikation der van Gies onschen Methode angewendet wurde, ließ sich Querstreifung nachweisen. Einmal deutlich ausgeprägt gefunden, kann man sich durch Vergleiche und Übung vom Vorhandensein zahlreicher quergestreifter Fasern mit undeutlicher bis zu fehlender Querstreifung überzeugen. Diese Methode sei jedermann, der sich mit ähnlichen Fragen beschäftigt, empfohlen (Färben mit Eisenhämatoxylin Weigerts, Nachfärben mit van Giesons Säurefuchsinpikrinsäure, rasches Entwässern); im Prinzip handelt es sich wie bei Kolisko um Pikrinsäurefärbung der Muskelfasern, doch hat sie vor dem Pikrokarmin den Vorteil konstanterer Resultate und rascherer Handhabung. Daß es nicht Zufall war, sondern einzig an der Methode gelegen hat, konnte man daraus ersehen, daß wir bei Serienschnitten aus einzelnen Partien bei Hämatoxylin-Eosin und anderen Färbungen stets 
vergeblich nach Querstreifung suchten, obwohl parallele Stellen mit ran Gieson solche deutlich und reichlich ergaben. (Wie ich nachträglich einem Referate über die Sitzung des naturhistorisch-medizinischen Vereins Heidelberg vom 10. Mai 1904 in der Münchener med. Wochenschrift 1904 Nr. 33. ersehe, hat auch Rosthorn die van Giesonsche Färbung, und zwar zur Differenzialdiagnose zwischen Myom und Myosarkom empfohlen.)

Die charakteristische Form der Kerne der Muskelbänder ist die elliptische, an Größe die der Kerne der Sarkomzellen übertreffend, etwa $14 \mu$ lang, $9 \mu$ breit. Hänfig findet sich im Kern ein Kernkörperchen oder mehrere Chromatinkörnchen. Die Lage der Kerne ist bei den deutlichen Fasern - und nur bei solchen läßt sich über die Angehörigkeit des Kernes sprechen, da bei den sich verzweigenden Fibrillen Täuschungen mit den dazwischen gelagerten Kernen der Sarkomzellen nioht auszuschließen sind - eine oberflächliche, also dem Fibrillenbündel anliegende; auch an Querschnitten fanden wir nur selten Bilder von Bündeln mit eingeschlossenen Kernen; diese sind dann selten zentral, sondern in einer peripheren Zone des Fibrillenbündels gelegen. Die Angaben der Autoren über das Verhältnis der Lagerung der Muskelkerne in Rhabdomyomen lauten verschieden. Bei den Myosarkomen (Adenosarkomen) der Niere, die eine verwandte Mischgeschwulstgruppe darstellen, fand sie Ribberti ${ }^{1}$ ) vorwiegend im Innern, Marchand ${ }^{2}$ ) an der Oberfläche. Zweifellos können dieselben in den Tumoren, entsprechend den verschiedenen Entwicklungsstufen der Muskelfaser, analog der embryonalen Entwicklung, wo sie zunächst zentral liegen und später zur Peripherie auswandern, einmal die, einmal eine andere Lage einnehmen. Auch Kolisko findet die Kerne bei der Bänderform am Rande wie wir. Es ist auch diese Bündelform als ein relativ vorgeschrittenes Stadium des sich herausdifferenzierendẹn Muskelgewebes zu nennen, gegen-

1) Ribbert, Beiträge zur Kenntnis der Rhabdomyome. Dieses Archiv, Bd. 130, 1892.

2) Marchand, Fall von Myosarcoma striocellulare der Niere. Dieses Archiv, Bd. 73, 1878. 
über den Spindelzellenformen, unả auch der Umstand, daß unsere Bänder sich am Querschnitte solid erweisen, für ein späteres Stadium, da Hohlgebilde, welche Ribbert oft beobachtete (wo die Fibrillenmasse einen nur mit undifferenziertem Protoplasma gefüllten Raum begrenzte), embryonal ebenfalls einer früheren Entwicklungsstufe angehören. Die Anwesenheit mehrerer Kerne bei der Länge der Bänder lag auf der Hand, ließ sich jedoch nirgends einwandsfrei zeigen.

Die Frage nach Vorhandensein von Sarkolemma bei Muskelfasern in Geschwülsten läßt sich an der Hand unserer Präparate im positiven Sinne beantworten; man beobachtet an einigen Querschnitten, daß die Fibrillenbündel daselbst von einer feinen Hülle umscheidet sind, die nicht etwa einem Bindegewebsseptum entspricht. Auch Hauser fand sie bei seinen Muskelfasern, während z. B. Ribbert ein Vorkommen von Sarkolemma negiert. A priori ist das Vorhandensein der Sarkolemmabildung bei einer vorgeschritteneren Entwicklungsstufe des Muskelgewebes auch theoretisch naheliegend.

Außer der Bandform finden sich an Stellen, wo quergestreifte Muskulatur nur spärlich vorkommt, ganz vereinzelte, rundliche Gebilde mit an Gewebe derselben erinnerndem Protoplasma mit einem oder mehreren in der Mitte gelegenen Kernen (bei 1 beschrieben); ihre Tinktion sprach für Muskulatur, doch vermag ich mich über ihren näheren Charakter bei ihrer Seltenheit nicht zu äußern.

An Zupfpräparaten gelingt der Nachweis der Querstreifung schwerer, und wir konnten das Verhalten derselben nicht verfolgen, da die Querstreifung nur in dickeren Bündeln noch zu konstatieren war, während sie in verkleinerten Elementen nicht mehr sichtbar wurde.

Neben den quergestreiften Muskelfasern ist das charakteristischste Gewebe für die Zusammensetzung dieser Geschwülste das aus Rund- und Spindelzellen bestehende Geschwulstgewebe, welches wir der Kürze halber hier Sarkomgewebe nennen. Auf die Gruppierung der Rund- und Spindelzellen zu Alveolen innerhalb eines faserigen Stromas, welche ein den Krebsnestern nicht unähnliches Bild liefert, wurde bereits öfters hingewiesen. In der Beschreibung eines Falles von Cervixsarkom äußert sich 
Kunert: ${ }^{1}$ ) Der Tumor erweist sich in seinen einzelnen Partien als ein aus dicht gelagerten, großen Rundzellen zusammengesetztes Gewebe. Eigentümlich ist hierbei die Anordnung der Rundzellenmasse in Form von zusammenhängenden Inseln, die sich durch eine dichte Anhäufung von Zellen an der Peripherie von dem umgebenden, mehr lockeren, spindelzelligen, teils auch faserigen Gewebe auffallend stark absetzen und so auf den ersten Blick Krebskörper vortäuschen. Ähnlich spricht Pf annenstiel ${ }^{2}$ ) von Herden, Nestern oder Zügen von Sarkomzellen inmitten normalen Bindegewebes, und W ilm s scheut sich nicht, einen Tumor Gangh ofers ${ }^{3}$ ) bei einem achtjährigen Mädchen, welcher mikroskopisch als alveoläres, von Cervixdrüsen ausgehendes Karzinom von Chiari diagnostiziert wurde, wegen der sonstigen Beschaffenheit als hierher gehörig anzuerkennen.

Auch bei unserem Tumor (Fig. 3, Taf. XI) findet sich dieses Verhalten sehr deutlich ausgeprägt, und dürfte die alveoläre Zelllagerung bei solchen Mischgeschwülsten nicht ohne diagnostisches Interesse sein und auch eventuell klinische Bedeutung besitzen. Da sich solche Mischgeschwülste aus anfänglich angeblich uncharakteristischen Polypen entwickeln, wäre das beschriebene Verhalten bei Beurteilung von Probeexcisionen von Wichtigkeit, um so mehr da es häufiger vorkommt und bisher nicht genügend gewürdigt wurde. Wie die Beschreibung eines Wilmsschen Falles zeigt, wohnt solchen Geschwülsten schon im Anfangsstadium der Entwicklung dieser bestimmte Bau inne, welcher sie sehr oft von einem gewöhnlichen Polypen zu unterscheiden gestattet. Dies wäre bei der tristen Prognose dieser Geschwülste zwecks frühzeitiger Operation von Wichtigkeit, da dieselben zumeist in ihrem Beginne von den Untersuchern für gewöhnliche benigne Polypen gehalten wurden.

Die die einzelnen Zellnester zusammensetzenden Zellen haben bei flüchtiger Betrachtung den Charakter von Rundzellen, mit

1) Kunert, Sarcoma uteri. Arch. f. Gynäkologie, Bd. 6, 1874.

2) Pfannenstiel, Das traubige Sarkom der Cervix uteri. Dieses Archiv, Bd. 127, 1890.

3) Zit. nach Pick, Sarkome des Uterus und der Vagina. Arch. f. Gynäkol., Bd. 46, 1894. 
rundlichen oder kantigen, viel seltener länglichen chromatinreichen Kernen. Wir überzeugten uns aber an sehr dünnen Schnitten und Stellen, wo die Zellen durch Risse im Präparat auseinandergewichen sind, daß diese zumeist als Rundzellen angegebenen Zellen nur zum kleinsten Teile Rundzellen entsprechen, sondern ihr Protoplasma fast stets radiäre, schwanzartige Fortsätze aussendet, somit die Form der Zellen eigentlich eine sternförmige ist (Fig. 4, Taf. XI). Hie und da bemerkt man öfters und bei gewöhnlicher Betrachtung, daß die Zellen in einer Richtung zu Zügen geordnet verlaufen, und so in den Nestern Übergänge von der vermeintlichen Rundzellenform zur Spindelzelle bestehen; tatsächlich sind sie jedoch auch hier mehr oder weniger sternförmig. Dies seheint uns nicht unwichtig für die Beurteilung des öfter behandelten Themas, ob das schleimige Gewebe solcher Geschwülste einem oedematösen Bindegewebe (Pfannenstiel bei Cervixsarkomen) oder echtem Schleimgewebe entspreche, und kann für die letztere Auffassung verwertet werden, da die bereits ursprünglich sternförmigen Zellformen für eine weitere Entwicklung zum Schleimgewebe durch ihre Form einen Fingerzeig abgeben - was ja für einige Fälle durch die Mucinreaktion (mit Essigsäure feinkörniger Niederschlag) direkt bewiesen wurde (Marchand).

In unseren Bildern konnte man nirgends von einem schleimigen Gewebscharakter sprechen, die Zellen wichen an keiner Stelle auffallender auseinander, sondern lagen entweder zu Nestern geformt im Stroma, oder infiltrierten dasselbe im Vereine mit den übrigen Tumorelementen. Auch die derbere Konsistenz ließ in dieser Beziehung schon äußerlich unsere Geschwulst von den weichen, polypenartigen unterscheiden.

Gegen die Stauungserklärung der weichen Beschaffenheit dieser Geschwülste ist nicht unwesentlich, daß z. B. in unserem Falle für eine solche disponierende Ursachen genügend vorlagen, da der Tumor an der Cervix als Stiel vor dem Genitale pendelte; und doch zeigt sich nirgends oedematöses resp. schleimiges Gewebe.

In der Regel begegnet man Angaben, daß der Bau der alveolären Stellen ein derartiger ist, da $\dot{B}$ sich dabei Rund- und Spindelzellen mit einander mischen, i. e. die Spindelzellen zu 
Zügen geordnet Rundzellen alveolär einschließen. Dieses Verthalten konnte ich nicht beobachten. Unsere Zellen, die je nach der Form der Kerne and gegenseitiger Lagerung mehr oder weniger das Aussehen von Rund- und Spindelzellen darboten, waren vom Bindegewebe umscheidet, $d$. h. die Zellnester befanden sich im Stroma, welches rein faserig, zellarmem, fibrillärem Bindegewebe gleichend, aussah; die darin vorkommenden Zellen, von denen nur die schmalen Kerne zu beobachten waren, bezeichne ich füglich als Bindegewebszellen. Eine ebensolche Beschaffenheit findet sich auch in den Abbildungen der meisten Autoren (Pfannenstiel, Wilms) und dürfte dem gewöhnlichen Verhalten entsprechen.

Angenommen wird, daß bei den Tumoren von schleimigem Charakter das zwischen den Zellnestern gelegene Stroma sich zum Schleimgewebe differenziert. Nach unseren Zellbildern ist näherliegend, daß, wenn in solchen Geschwülsten ein Bestandteil myxomatös wird, dies das Gewebe der Nester betrifft, weniger und sekundär das Stroma. In diesem Sinne sind die in den Nestern vorkommenden Hohlräume in Abbildungen Pfannenstiels zu verwerten, und es scheinen uns also an der Bildung des Schleimgewebes nur die Nester aktiv teilzunehmen, was ja auch der. Wilmsschen Hypothese der Differenzierung aus den Tumorzellen entspricht.

Wir würden uns somit die einzelnen Stadien der Entwicklung der Geschwulst im Anschlusse an die Wilmssche Entstehungstheorie so vorstcllen, daß zunächst durch Wucherung des supponierten embryonalen, in seiner Entwicklung zurückgebliebenen Keimes ein Gewebe aus mehr rundlichen oder länglichen Sternzellen entsteht, welches die Eigenschaft hat, sich innerhalb des ursprünglichen Mutterbodens in Nester bezw. Sänlen zu ordnen. Diese Zellen besitzen die Eigenschaft, sich myxomatös umzuwandeln. Im vorgeschritteneren Differenzierungsgrade, wobei anders geartete Gewebe in ihm auftreten (quergestreifte Muskelfasern, Knorpel im Schleimgewebe usw.), wird die Gruppierung der Zellen zu Nestern immer undeutlicher und einer diffusen Gewebsinfiltration gleichend. Wir vermissen z. B. stets in Partien, in welchen sich ausgesprochene Nesteranordnung der Zellen vorfand, eine reichlichere Anzahl der 
quergestreiften Muskelelemente, und können insbesondere die Form der ausgesprochenen Muskelbänder nicht nachweisen, während man auf ihre reichliche Anwesenheit bei genauerer Musterung stets dort rechnen kann, wo das Geśchwulstgewebe einem diffusen Infiltrate ähnlich aussieht. Im relativ höchsten Grade, mit den best entwickelten Muskelfasern, wie in Fig. 4, ist auch der Charakter einer gleichmäBigeren Infiltration verschwunden; man sieht die sich verwebenden Züge und Bündel von Bindegewebe und Muskelgewebe, wo nur der Zellreichtum an die ursprüngliche Geschwulstnatur mikroskopisch erinnert. Die beiden Gewebsarten, quergestreifte Muskulatur und Geschwulstzellen, stehen in gegenseitiger Korrelation, indem stets ein besonderer Entwicklungsgrad der Muskelfasern einer besonderen Form der Geschwulstzellenanordnung entspricht. Während sich die alveoläre Zellgruppierung mit allmählicher Differenzierung mehr und mehr auflöst, steigt die Anzahl und auch der Entwicklungsgrad der Muskelfasern. Diese Beziehung konnte bei unserer Geschwulst jedoch nur für diese zwei Gewebe beobachtet werden. Mit den Geschwülsten Hausers oder Koliskos verglichen, zeigt unsere im ganzen einen höheren Differenzierungsgrad.

Unter anderen vorkommenden Elementen sind die glatten Nuskelfasern zu nennen; die Reichlichkeit ihres Vorkommens reicht nirgends an die der quergestreiften heran. Man findet sie am häufigsten in Form von Längs- oder Querbündeln, die häufig parallel mit dem Verlaufe größerer Gefäße, oder aber, wie man der Schilderung der Cystenwand im. Innern des Tumors entnimmt, in der Richtung der den Hohlraum begrenzenden Gewebe (tangential) angeordnet sind, also der an einzelnen Stellen gegebenen Verlaufsrichtung der Gewebe überhaupt folgen, ohne eine Verflechtung zu zeigen, wie die quergestreiften Fasern. Die glatten Muskelzellen sind als solche überall leicht zu erkennen; Verwechslungen beider scheinen mir, sofern das Gewebe einen einigermaßen bestimmbaren Charakter hat, ziemlich ausgeschlossen, auch konnte ich keinerlei Utbergänge der glatten in quergestreifte finden. Die Bündel, welche sich oft bis zur Länge von $2 \mathrm{~mm}$ verfolgen lassen, bestehen aus einander anliegenden, glatten Muskelzelleu 
von charakteristischer spindelförmiger Gestalt. Dieselben sind im Verlaufe ebenfalls gewellt, wie die quergestreiften Bänder, doch hat ihre Schlängelung einen feineren, regelmäßigen Charakter (ähnlich wie fibrilläres Bindegewebe), während sie bei den letzteren mehr unregelmäßigen Verbiegungen ähnelt. Es ist auch ihr gegenseitiges Mischungsverhältnis mit normalem Bindegewebe ein viel innigeres als das der quergestreiften Muskelfasern. Ihr Verhalten gegenüber den eigentlichen Tumorzellen ist einigermaßen auffallend: während die quergestreiften Elemente äberall zu denselben in bestimmter Beziehung stehen und überall von denselben begleitet sind, also ihre Entwicklung mit der Veränderung der Sarkomzellen parallel geht, finden sich die glatten Muskelfasern nirgends (ausgenommen natürlich die Gefäßmuskulatur, von der jedoch hier nicht die Rede ist) mit den Tumorzellen vergesellschaftet. Im Gegenteil, in unseren Präparaten fehlten stets in Umgebung von Tumorzellen die Bündel glatter Muskelfasern vollständig, sie wurden vom gewöhnlichen areolären Bindegewebe begleitet, und boten derartige Stellen, trotzdem sie z. B. nicht im Stiele der Geschwulst, sondern mehr in der Mitte gelegen waren, für sich betrachtet, keine Anhaltspunkte für Tumor. Auch war das Aussehen der glatten Muskulatur nie das eines unfertigen, in Differenzierung begriffenen Gewebes, wie das der quergestreiften. Jedenfalls ist bei der Annahme, daß sich aus den embryonalen Keimen glatte und quergestreifte Muskelfasern entwickeln, die Beobachtung, daß die Beziehungen beider zu Sarkomzellen so ungleichmäßig sind, nicht ohne Interesse, doch lassen sich auf Grund eines Falles keine Schlüsse ziehen, ob und wie die glatte Muskulatur an der Bildung einer solchen Geschwulst aktiv partizipiert. Nach Wilms wächst alles Gewebe im Tumor, selbst Stroma mit Gefäßen, glatte und quergestreifte Muskelfasern, Knorpel usw. aus dem Geschwulstkeime selbst, nicht aus dem Mutterboden hervor. Wie man in unseren Bildern für die aktive Beteiligung des Stromas bei der Schleimgewebsbildung und auch sonst keinen Anhalt findet, so daß wir glauben, daß dasselbe, wenn nicht ganz, so doch zum größten Teile dem Mutterboden angehört, ähnlich scheinen dieselben auch für die Nichtbeteiligung des Tumors bei 
Entstehung der glatten Muskelfasern zu sprechen. Allerdings kommen in den ihrer Zusammensetzung nach nahe verwandten Mischgeschwïlsten der Niere glatte Muskelfasern vor neben quergestreiften, welche beide hier dem Mutterboden entstammen können. Jedenfalls treten sie in Geschwülsten des weiblichen Geschlechtsschlauches zu Tumorzellen in wenig Beziehung, und lassen sich die bezüglichen Literaturangaben bzw. Bilder selten für Entstehung im Tumor selbst verwerten.

Sehr häufig wurden bei Sarkomen der Cervix Drüsen beobachtet. In unserem Falle finden sich dieselben in verschiedenen Stellen des Präparates zerstrent und sind nicht nur in den Grenzpartien der Geschwulst, sondern hie und da auch mehr zentral gelegen. Dieselben stellen im Querschnitte rundliche oder gebuchtete Räume dar, welche mit hohem, einreihigem Cylinderepithel ausgestattet sind. Gegen das Stroma sind sie wie normale Cervixdrüsen durch flache Bindegewebszellen getrennt und weichen überhaupt vom normalen Bane nicht ab. Wenn auch von Kahlden ${ }^{1}$ ) bei Besprechung dieser Drüsen bei einer hierher gehörigen Cervixgeschwulst Pfannenstiels „neugebildete Drüsenräume für wahrscheinlich hält", so sind sie sicher als dem Mutterboden angehörend zu betrachten, schon deshalb, weil sie bei Mischgeschwülsten, die entfernter von der Uterusgrenze entspringen, stets vermißt werden und in ihrem Bane vollkommen mit dem der Cervixdrüsen übereinstimmen. Bekanntlich wurden Bilder der Cervixtumoren, wo sich Drüsen im Geschwulstgewebe fanden, als Adenosarkome (Mundé) gedeutet im Sinne einer aktiven Betätigung des Drüsenkörpers am Geschwulstbaue. In der Tat findet man öfter Bilder, wo inmitten eines bindegewebigen Stromas die schön entwickelten, wohlerhaltenen Drüsenquerschnitte von einer Tumorzelleninsel umschlossen sind. Die Ansicht $\mathrm{Mu}$ u dés ist allgemein verlassen, obwohl es auffällig ist, dab bei diesen Tumoren die epithelialen Elemente inmitten des Geschwulstgewebes sehr lange erhalten bleiben. Neben den Drüsenkörpern ist es in unserem Falle die durch die ganze Tumordicke durchziehende, erhaltene und ihres Epithels nicht beraubte Cervix (obwohl das Epithel von

1) von KahIden, Das Sarkom des Uterus. Zieglers Beiträge, Bd. 14. 1893. 
Tumorzellen dicht eingeschlossen ist); ähnlich ist das Erhaltensein des eylindrischen Oberflächenepithels bei den traubenmolenartigen Cervixsarkomen charakteristisch und gewöhnlichen Sarkomen nicht in diesem Maße eigen. Kahlden spricht sich diesbezüglich dahin aus, „daß da, wo überhaupt die Sarkomwucherung so weit in die Schleimhaut vordringt, daß sie die Drüsen vollständig einschließt, diese zusammengedrückt werden und sehr bald vollständig verschwinden .... Etwas derartiges (i. e. unveränderte Drüsenlumina im Geschwulstgewebe), wo sogar irgendwelche Kompressionserscheinungen von seiten des wuchernden Geschwulstgewebes fehlen, wird man in einem Sarkom niemals finden". 'Jedenfalls spricht die Eigentümlichkeit der langen Persistenz der epithelialen Bildungen im Geschwulstgewebe nicht allein für einen besonderen Charakter dieser von den Sarkomen zu trennenden Mischgeschwülste, sondern die häufigen Drüsenbefunde stehen mit der Annahme der Entstehung des Stromas nur aus den Tumorzellen (gegenüber Abstammung vom Mutterboden) nicht im Einklange.

Hauser erwähnt bei Schilderung seines Falles eigentümliche zerstreute Haufen großer epithelähnlicher Zellen, welche an der Grenze des Tumors teils noch im Bindegewebe der Scheidenschleimhaut, teils in der Geschwulstmasse bereits eingebettet lagen. Er beschreibt sie als ,große, bis zu $0,045 \mathrm{~mm}$ im Durchmesser haltende, polygonale Zellen mit scharf konturiertem Protoplasma, und großem, bläschenförmigem, etwas hellerem Kern, welcher ein dunkles Kernkörperchen einschließt. Die Zellen liegen in der Mitte der Zellhaufen dicht aneinander und bilden ein unregelmäßiges Mosaik, während dieselben gegen die Peripherie hin mehr und mehr schmale Zwischenräume zwischen sich lassen, in welchen stark gewundene, glatte Muskelfasern verlaufen." Außer von Hauser wurden in solchen Tumoren ähnliche Bildungen nicht beobachtet, und wird deren epitheliale Natur von Wilms nach Durchsicht der Präparate Hausers angezweifelt. - In unseren Präparaten fanden sich Gebilde, die an die Schilderung Hausers erinnern, die aus großen epithelartigen Zellen, mit bläschenförmigem Kern und deutlichen Kernkörperchen bestehen. Sie zeigten in der Mitte dichtere Anordnung, wichen in der Peripherie mehr 
auseinander, und ihre Leiber sind daselbst wie in Fenster eines Fasernetzes (das dem Hauserschen glatten Muskelzellenbelag derselben vielleicht entsprechen würde) eingelagert. In ihrer Mitte fand sich nicht selten ein rote Blutkörperchen enthaltendes Gefäßchen. Ihre Lokalisation war analog Hauser die Grenze des Tumors gegen das Schleimhautgewebe der Portio bzw. Uterus, und fanden sich diese Bildungen regelmäBig vergesellschaftet mit den Querschnitten der Drüsen. Die längere Untersuchung machte es wahrscheinlich, daß beide Bildungen zusammen in Beziehung stehen, und daß diese Gebilde Stellen, wo früher Drüsen gelegen haben, entsprechen. Man findet nicht selten um die Drüsenquerschnitte das Bindegewebe sich areolär in Form von circulären Fasern lagern, zwischen welchen zunächst Leukocyten, Bindegewebszellen sowie auch einzelne kleinste Gefäßchen vorkommen. Besonders in der Adventitia solcher Gefäßchen zeigen die Zëllen sehr deutlich die Form der beschriebenen epitheloiden; wenn auch zunächst kleiner, bestehen doch allmähliche Größenunterschiede bis zu solchen von $40 \%$ Durchmesser in den kugeligen Gebilden. Dort wo sich die Gefäßhehen einem Drüsenquerschnitte anlagern, wird derselbe eingedrückt, indem diese Zellen das Drüsenepithel gegen die Mitte schieben. Bei Weiterwachstum dieses Gewebes reröden und verschwinden endlich die Drüsen vollständig, und wird der früher von ihnen innegehabte rundliche Hohlraum von den erwähnten Bildungen eingenommen. Die in Frage kommenden Zellen, welche übrigens vereinzelt spärlicher in der Umgebung der rundlichen Gebilde liegen, scheinen mir den Granulationsgewebs - (und weiter Bindegewebs-) Zellenformen zuzurechnen and reaktiven Ursprungs zu sein - schon aus dem Begleiten der Leukocyten zu schließen. Bei der regressiven Metamorphose des Gefäßchens bilden sich um die Zellen die homogenen, mit $\vee$ an Gieson gelb färbbaren Umscheidungen, die Mosaik.

Ob die Gebilde in Hausers Präparaten unseren vollkommen gleich zu halten sind, und ob die normalerweise in der Scheide ganz vereinzelt vorkommenden Drüsen oder vielleicht etwas anderes zu ihrer Entstehungserklärung herbeigezogen werden könnte, vermag ich nach den Abbildungen nicht zu entscheiden. 
Beziehungen des elastischen Gewebes zum Tumor fanden sich keine; in versehiedenen Partien der Geschwulst, in der Schleimhant, im Bindegewebsstroma und in den Gefäßen konnten mittels spezieller Färbung (Weigert) elastische Fasern nachgewiesen werden, doch nur in solchem Verhältnisse, wie normaliter. An Stellen, wo spezifische Tumorelemente (Rundzellenhaufen, Muskelgewebe) vorkamen, fehlten elastische Fasern stets. - Das Vorhandensein von Knorpel war nach dem mehr vaginalen (Portio) als cervikalen Sitze der Geschwulst von vornherein nicht $\mathrm{zu}$ erwarten.

Am Schlusse noch über die Systematik solcher Tumoren. Man hat bei dieser Geschwulstgruppe, die mit als Sarkom angesehen wurde, eine Scheidung der Vaginalsarkome von den Cervixsarkomen, ferner eine prinzipielle Trennung der des kindlichen Alters von denen der Erwachsenen durchführen wollen, und wurde hierzu sowohl Klinisches, als Anatomisches als Stütze benutzt. Nach Rosthorn, ${ }^{1}$ ) welcher die diesbezüglichen Merkmale der Autoren präzisiert, zeigen die Tumoren der Cervix traubige Form, sind ausgesprochen gestielt, von polypoidem Charakter und werden einzeln mit Blasenmolen verglichen; mikroskopisch findet sich Schleimgewebe, event. mit quergestreiften Muskelfasern und Knorpel, vor. Ähnlich verhält sich die Gruppe der kindlichen Scheidentumoren, während die Geschwülste der Scheide Erwachsener teils als starre Infiltration, teils als umschriebene Tumoren auftreten und fremdes Gewebe nicht aufweisen. Klinische Unterscheidungsmerkmale fußen auf dem Umstande, daß die Neubildung der Scheide bei Erwachsenen ziemlich begrenzt bleibt, während sie bei Kindern infiltrativ an die Scheide, Blasenschleimhaut und Ureterenmündung fortschreitet und dadurch rasch zum Exitus führt. (Neben der Beschreibung der in der Cervix und Vagina vorkommenden Sarkome führt R. einen Fall von Sarkom der Vaginalportio an, welcher mit Rücksicht auf sein Verhalten gegenüber dem Einteilungssehema angeschlossen wird.)

Eine scharfe Trennung der besprochenen Tumoren halte ich für nicht möglich. Alle diese Geschwïlste, i. e. die Misch-

1) Rosthorn, Zur Kenntnis des primären Sarkoms der Portio vaginalis und der Vagina. Wiener Klin. Wochenschrift 1889, Nr. 38. 
geschwälste, besitzen einen nahe verwandten Charakter, welchen auch die der Niere teilen. Wenn man sich von dem alten Schema der gutartigen und bösartigen Tumoren und der Einteilung letzterer in Sarkome und Karzinome emanzipiert und die Gruppe der zusammengesetzten Tumoren (Mischgeschwülste) als selbständig akzeptiert, wenn man also die eigentlichen Sarkomformen des Genitales ausscheidet (die nam. als Angiosarkome, Spindelzellensarkome etc. beschrieben wurden), so kann man höchstens bei diesen, ihrer Zusammensetzung und Histogenese nach gleichen Tumoren von versčhiedenen Typen der Form ihres Auftretens gegenwärtig sprechen. Die eine Form wäre die papilläre, d. i. der vielfachen Polypen und Trauben, die zweite die des umschriebenen Knotens; der erste Typus würde mehr den cervikalen, der zweite mehr den vaginalen Sarkomen von früher entsprechen. Zwischen beiden gibt es allmähliche Ubergänge. Einer scharfen Trennung würde z. B. unser Tumor Schwierigkeiten bereiten, da er vom grobanatomischen Standpunkte vaginalen Charakters, i. e. der Portio, angehört (wenn man Sarkome der Portio von denen der Cervix scheiden will, wofür man klinisch das Verbleiben der Scheidengewölbe in situ bei seinem Prolabieren verwerten kann, da bei der Schrôder schen Dreiteilung des Gebärmutterhalses bei der Senkung der Cervixaffektion die Scheidengewölbe mitgehen) und mit den Scheidensarkomen Erwachsener die Form und das Alter der Trägerin gemeinsam hat, während sein komplizierter Bau ihn wieder den kindlichen Tumoren näherstellt und das Vorhandensein der Cervixdrüsen ihn in die Cervixgruppe einreihen läßt.

Auch bei dem Rosthornschen Falle eines Portiosarkoms braucht das Fehlen der quergestreiften Maskulatur das Unterscheidungsmerkmal nicht abzugeben; das Fasergewebe seines ersten mikroskopischen Bildes schließt die Möglichkeit des Vorliegens von Querstreifung, welche eventuell bei geeigneten Färbungen erst vortreten würde, nicht ganz aus. Übrigens bereitete auch die Einteilung des ersten, historisch gewordenen Falles von Rhabdomyosarkom der Vagina (KaschewarowaRudnewa) einer 15 jährigen Person dem bisherigen Schema Schwierigkeiten - die Geschwulst, welche zunächst schleim- 
polypenartig war, wuchs nach Exstirpation als Knollen und wurde teils den Sarkomen der Kinder, teils denen der Erwachsenen zugezählt. Warum in dem einen Falle eine mehr weiche, schleimige Konsistenz, im anderen derbere resultiert, ist nicht mit Sicherheit anzugeben; Wilms hält für erstere das freie, unbehinderte Hineinwachsen in eine präformierte Höhle für wesentlich. Dasselbe war aber bei unserer Geschwulst ebenfalls vorhanden. Nachdem derbe Tumoren im allgemeinen der Portio und der Scheide der Erwachsenen, weiche (polypöse) der Kinderscheide und Cervix entsprechen, scheint die feste oder lockere Beschaffenheit des Mutterbodens nicht ohne Bedeutung zu sein. Die eigentliche Ursache der Geschwulst, der verlagerte embryonale Keim im sinne Wilms, bleibt doch überall relativ gleich.

Auf die ziemliche Seltenheit unserer Beobachtung besonders hinzuweisen, habe ich für nicht notwendig gehalten.

\section{Erklärung der Abbildungen auf Taf. XI.}

Fig. 1. Die Lage des Tumors am schematischen Beckendurchschnitte. Fig. 2. Seitenansicht des exstirpierten Tumors in etwa $\frac{3}{4}$ seiner wirklichen Größe. Die mit Plattenepithel bedeckte Oberfäche weißlich vortretend; die trichterförmige Einziehung des oberen Randes entspricht der Abtrennungsstelle vom Uterus.

Fig. 3. Oberflächliche, mit Plattenepithel bedeckte Partie der Geschwulst bei schwacher Vergrößerung. Alveolärer Bau; Tumorgewebe in Nesterform angeordnet.

Fig. 4. Die Zelltypen der einzelnen Nester der vorherigen Figur bei Immersion an sehr dünnen Stellen.

Fig. 5. Zentral gelegener Geschwulstanteil bei Immersion; im Gewebe reichlich Bündel quergestreifter Muskulatur und Tumorzellen; die im van Gieson-Präparate mit Säurefuchsin tingierten Bindegewebsfibrillen als schwarze Streifen dargestellt. 
है
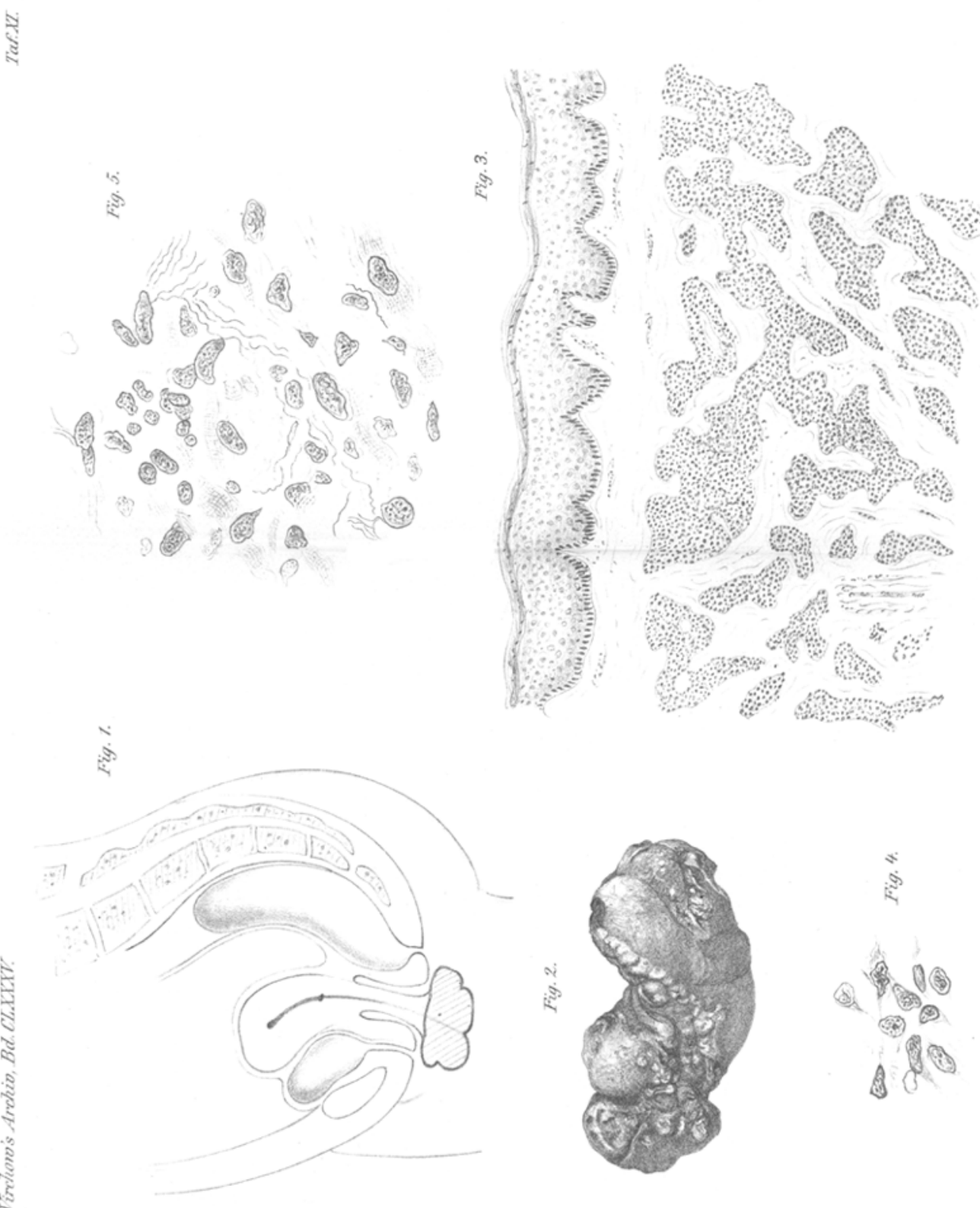